\title{
Lipin 1 Is Involved in the Pathogenesis of Diabetic Encephalopathy through the PKD/Limk/Cofilin Signaling Pathway
}

\author{
Min Xie, ${ }^{1,2}$ Meijian Wang, ${ }^{1}$ Wei Liu, ${ }^{3}$ Min Xu, ${ }^{1}$ Pan Shang, ${ }^{1}$ Dongqing Jiang, ${ }^{1}$ Liping Ju, \\ Fei Wu, ${ }^{1}$ Aili Sun, ${ }^{1}$ Shuyan Yu, ${ }^{4}$ Xianghua Zhuang ${ }^{1},{ }^{1}$ and Shihong Chen $\mathbb{1}^{1}$ \\ ${ }^{1}$ Department of Endocrinology and Metabolism, The Second Hospital, Cheeloo College of Medicine, Shandong University, \\ Jinan 250012, China \\ ${ }^{2}$ Department of Endocrinology and Metabolism, Binzhou Medical University Hospital, Binzhou 256603, China \\ ${ }^{3}$ Department of Encephalopathy, The Second Affiliated Hospital of Shandong University of Traditional Chinese Medicine, \\ Jinan 250001, China \\ ${ }^{4}$ Department of Physiology, School of Basic Medical Sciences, Cheeloo College of Medicine, Shandong University, Jinan 250012, China
}

Correspondence should be addressed to Xianghua Zhuang; sd73095760653@163.com and Shihong Chen; chenshihong26@163.com

Received 19 May 2020; Revised 3 August 2020; Accepted 1 September 2020; Published 17 October 2020

Academic Editor: Nadja Schroder

Copyright (c) 2020 Min Xie et al. This is an open access article distributed under the Creative Commons Attribution License, which permits unrestricted use, distribution, and reproduction in any medium, provided the original work is properly cited.

\begin{abstract}
Diabetic encephalopathy is a type of central diabetic neuropathy resulting from diabetes mainly manifested as cognitive impairments. However, its underlying pathogenesis and effective treatment strategies remain unclear. In the present study, we investigated the effect of Lipin1, a phosphatidic acid phosphatase enzyme, on the pathogenesis of diabetic encephalopathy. We found that in vitro, Lipin1 exerts protective effects on high glucose-induced reductions of PC12 cell viability, while in vivo, Lipin1 is downregulated within the CA1 hippocampal region in a type I diabetes rat model. Increased levels of Lipin1 within the CA1 region are accompanied with protective effects including amelioration of dendritic spine and synaptic deficiencies, phosphorylation of the synaptic plasticity-related proteins, LIM kinase 1 (p-limk1) and cofilin, as well as increases in the synthesis of diacylglycerol (DAG), and the expression of phosphorylated protein kinase D (p-PKD). These effects are associated with the rescue of cognitive disorders as shown in this rat model of diabetes. In contrast, knockdown of Lipin 1 within the CA1 region enhanced neuronal abnormalities and the genesis of cognitive impairment in rats. These results suggest that Lipin1 may exert neuroprotective effects involving the PKD/Limk/Cofilin signaling pathway and may serve as a potential therapeutic target for diabetic encephalopathy.
\end{abstract}

\section{Introduction}

Diabetes can result in chronic complications involving multiple systems. In some diabetic patients, varying degrees of cognitive deficits and even dementia may be present. Accordingly, the term "diabetic encephalopathy (DE)" was first proposed in 1950 to describe the cognitive impairments related to diabetes [1]. Such impairments in cognitive function, which can be revealed as declines in learning, attention, and spatial memory, comprise an important basis for the disability and death of these patients. However, the exact pathogenesis of $\mathrm{DE}$ is quite complex and remains unclear [2]. Therefore, a more detailed understanding of DE pathogenesis will be required in order to identify new approaches for the prevention and treatment of this condition.

Lipin1, a phosphatidic acid phosphatase enzyme, plays an important role in glycolipid metabolism [3] and participates in triacylglycerol biosynthesis through either a $\mathrm{Mg}^{2+}$ dependent phosphatidate phosphatase or as a transcriptional coactivator of genes in the oxidative metabolism of fat $[4,5]$. In the muscle, Lipin 1 can convert phosphatidic acid (PA) to diacylglycerol (DAG) to further activate protein kinase D 
(PKD) [6]. In addition, Lipin1 also appears to exert effects within the nervous system as demyelination is observed in Lipin 1 mutant mice, effects which appear to be mediated by a PA metabolic disturbance [7].

Previous work within our laboratory has revealed that the occurrence of diabetic peripheral neuropathy (DPN) in rats may be related to a decline in Lipin1 expression, while an upregulation in Lipin1 levels improves nerve conduction velocity and the pathological morphology observed within a diabetes mellitus (DM) rat model [8]. We have also found that cognitive impairments were present in $\mathrm{Lpin} 1^{\mathrm{fld}} / \mathrm{J}$ mice [9]. Whether Lipin1 may be involved in the pathogenesis of DE and, if so, the potential molecular mechanisms that underlie such effects have not been elucidated. Accordingly, the goal of this report was to address these issues. As an approach to accomplish this goal, we have conducted this study.

\section{Materials and Methods}

2.1. Animals. Male Wistar rats (6-8 weeks old) were obtained from the Shandong University Animal Center. All animal experiments were approved by the Animal Care and Use Committee of Shandong University and were conducted in accordance with the National Institutes of Health guidelines for the care and use of laboratory animals. Rats were maintained on a $12 \mathrm{~h}$ light/dark cycle at room temperature of $24 \pm 2^{\circ} \mathrm{C}$ in the animal laboratory of the Shandong University.

2.2. In Vivo Diabetes Model. For the in vivo model of diabetes, streptozocin (STZ, Solarbio, China), which was freshly prepared in cold $0.1 \mathrm{M}$ citrate buffer ( $\mathrm{pH} 4.5$, Solarbio, China), was injected intraperitoneally (i.p.) into rats using a single dose of $60 \mathrm{mg} / \mathrm{kg}$ body weight. Rats in the control group were injected with an equivalent dose of citrate buffer. Three days after this STZ injection, fasting blood glucose (FBG) levels (with a minimal fasting period of $8 \mathrm{~h}$ ) were determined. Levels $>11.1 \mathrm{mM}$ were established as the diagnostic criteria for T1DM in this rat model. Blood glucose values were monitored as achieved from samples collected from the tail veins of the rats. Blood glucose levels and body weights were determined at 1, 2, 4, 8, and 12 weeks after the STZ injection. All animal experiments were conducted in the Department of Physiology of the School of Basic Medical Sciences at Shandong University.

2.3. In Vivo Central Nervous System Treatment. Lentiviral up- and downregulation of the Lipin 1 and the blank vectors were constructed and encoded with the green fluorescent protein sequence (Jikai Gene Chemical Technology Co., Ltd.): Lv-Lipin1 $\left(1 \times 10^{9} \mathrm{TU} / \mathrm{mL}\right)$, Lv-Lipin1-Con $\left(2 \times 10^{9} \mathrm{TU} / \mathrm{mL}\right), \mathrm{Lv}$-Lipin1ShRNA $\left(7 \times 10^{8} \mathrm{TU} / \mathrm{mL}\right)$, and Lv-Lipin1ShRNA-Con $\left(1 \times 10^{9} \mathrm{TU} / \mathrm{mL}\right)$. The sequence of Lipin1ShRNA was caGCGAGTCTTCAGACACTTT. The sequence of Lipin1ShRNA-Con is TTCTCCGAACGTGT CACGT.

The rats were anesthetized with sodium pentobarbital $(40 \mathrm{mg} / \mathrm{kg}$, i.p.) and placed in a stereotaxic frame (Stoelting,
USA). The Lv-Lipin1ShRNA and blank vector was injected into WT rats (WT+Lv-Lipin1ShRNA and WT+Lv-Con groups). The Lv-Lipin1 and blank vector was injected into $\mathrm{DE}$ rats (DE+Lv-Lipin1 and DE+Lv-Con groups). These rats would then be tested for behavioral responses as described below. The lentovirus (Lv) injection was administered bilaterally to the hippocampal CA1 region (A/P, $-3.24 ; \mathrm{L}, \pm 2.5$; $\mathrm{D} / \mathrm{V},-2.45 \mathrm{~mm}$ relative to bregma). A total of $10 \mu \mathrm{L}$ was administered ( $5 \mu \mathrm{L}$ per side of hippocampus) at a flow rate of $0.15 \mu \mathrm{L} / \mathrm{min}$ with use of a $5 \mu \mathrm{L}$ microsampler (Gaoge, Shanghai, China). The needle remained at the injection site for $10 \mathrm{~min}$ and was then gently retracted. The wound was then sterilized and sutured. At 14 days after injection, the rats were anesthetized and slowly perfused with saline and $4 \%$ paraformaldehyde (PFA). The brain was carefully removed and placed in $4 \%$ PFA at $4^{\circ} \mathrm{C}$ overnight before undergoing graded dehydration. Frozen brain samples were cut into $30 \mu \mathrm{m}$ thick consecutive coronal sections and stored at $-20^{\circ} \mathrm{C}$ for the subsequent experiments.

\subsection{Behavioral Tests}

2.4.1. Open Field Test. In the open field test, the rats' movement within a novel environment was recorded, which can serve as a means to evaluate their activity and anxiety. Each rat was placed in the center of the open field box $(100 \times 100 \times 50 \mathrm{~cm})$ at the beginning of the test [10]. The floor of the apparatus was divided into 25 squares. The motion trajectory of each rat was recorded for 5 min using a computerized video system (SMART, Panlab, Spain). The time spent in the central squares and the number of crossings of the squares were then used to assess the degree of anxiety, with maintenance in the center squares and limited crossings indicating high levels of anxiety. After each open field test, the cage was carefully cleaned with $75 \%$ ethanol to eliminate any cues which could affect the behavioral responses of the following rats.

2.4.2. Novel Object Recognition (NOR) Test. The NOR test provides a means to evaluate the learning and memory ability of the rats. The task was divided into an adaptation, training, and testing period $[11,12]$. For the adaption period, the rats were placed into the apparatus at the same position and allowed to move freely for $5 \mathrm{~min}$. At $24 \mathrm{~h}$ after this adaption period, the training period was initiated. During the training period, two identical objects (A1 and A2) were placed symmetrically in the left and right corners of the test box. The time and frequency of exploratory behavior directed to the objects were recorded for $5 \mathrm{~min}$. After this training period, the rats were returned to their original home cage for a $1 \mathrm{~h}$ period. During the test period, the rats were again placed in the test apparatus, which now contained a (novel) B object in place of the A2 object. Similar to that of the training period, time and frequency of exploratory behavior directed to the objects ( $\mathrm{A} 1$ and $\mathrm{B}$ ) were recorded for $5 \mathrm{~min}$.

The criteria required for defining this as exploratory behavior directed to the objects consisted of direct contact with the object and/or sniffing or licking the object or being located within $2-3 \mathrm{~cm}$ from the object. After each rat 
completed the test, the box and objects were wiped with $75 \%$ alcohol to eliminate any cues which could affect the behavioral responses of the following rats. A cognitive function score was defined by a discrimination index $(\mathrm{DI})=($ novel object exploration time - familiar object exploration time)/ total exploration time.

2.4.3. Morris Water Maze (MWM). The MWM is a classic experiment for assessing spatial learning and memory function in rats [13]. A circular chamber $(120 \mathrm{~cm}$ in diameter and $60 \mathrm{~cm}$ in height) was divided into four equal quadrants and contained water with black food dye to ensure that the water was opaque. The depth of the water was $40 \mathrm{~cm}$, and the temperature was maintained at $22 \pm 2^{\circ} \mathrm{C}$. A platform $(13 \mathrm{~cm}$ in diameter and $38 \mathrm{~cm}$ in height) was placed in the center of quadrant three and was submerged to $2 \mathrm{~cm}$ underwater. Markers were positioned around the pool to help the rats locate the platform. The test consisted of four days of place navigation tests with four trials per day and one day of a spatial probe test. Rats within the various experimental treatments (as described above) were randomly placed in quadrant three and allowed to locate the platform within $60 \mathrm{~s}$ during the first four days of the navigation tests. If the rats failed to locate the platform, they were gently guided to the platform and allowed to remain on the platform for $10 \mathrm{~s}$. Escape latencies (60 s maximum) and times required to locate the platform were recorded. On the fifth day, the platform was removed, and each rat was allowed to swim freely for $60 \mathrm{~s}$. The time expended crossing over the area where the platform was located was recorded. All data and motion trajectories were recorded using a computerized video system (SMART, Panlab, Spain).

2.4.4. In Vitro Diabetes Model. For the in vitro diabetes model experiments, the PC12 (rat adrenal pheochromocytoma) cell line, purchased from the Shanghai Cell Bank of Chinese Academy of Science (Shanghai, China), was used. The cells were cultured in 25 mM Dulbecco's modified Eagle's medium under normal glucose (NG) concentrations (DMEM, Invitrogen, Carlsbad, CA, USA) with $1 \%$ penicillin/streptomycin (HyClone, USA) and 10\% fetal bovine serum (Lonsera, China) at $37^{\circ} \mathrm{C}$ in a humidified incubator containing $5 \%$ $\mathrm{CO}_{2}$. The medium was changed every two days. The cells were grown to approximately $80 \%-90 \%$ confluency. All cells were plated at an appropriate density in accordance with the needs of the experimental design.

2.5. CCK-8 Assay. A total of 4000 PC12 cells were seeded into 96-well plates and cultured for $12 \mathrm{~h}$ in complete medium. The cells were then cultured in DMEM containing 25, 50, 75, 100, 125 , or $150 \mathrm{mM}$ glucose for 24 or $48 \mathrm{~h}$. In addition, equal concentrations of mannitol were used as osmolarity controls to exclude the effect of osmotic pressure. Changes in cell viability were determined with the use of a cell counting kit-8 (Dojindo, Japan) according to the manufacturers' instructions. Cell proliferation was determined at $450 \mathrm{~nm}$ with the use of a microplate reader.

2.6. Virus Infection. The PC12 cells were seeded into 96well plates at a density of 4000 cells/well and cultured for $24 \mathrm{~h}$. Subsequently, lentiviral vectors (LVs; multiplicity of infection $=100)$, and a transfection enhancer, HitransG A (Jikai Gene Chemical Technology Co., Ltd.) were added to the cultured cells. The culture solution was changed after $8 \mathrm{~h}$.

2.7. Quantitative Real-Time Polymerase Chain Reaction (qRT-PCR). RNA from PC12 cells and tissues was extracted using the TRIzol reagent (Invitrogen, Carlsbad, CA, USA), while cDNA was generated using the primeScript RT reagent kit (TaKaRa, Otsu, Japan) in accordance with the manufacturers' instructions. qRT-PCR was performed using the SYBR $^{\circledR}$ Green Dye kit (TaKaRa, Otsu, Japan) and the BioRad CFX96 system. Relative gene expression levels were calculated using the classic $2^{-\Delta \Delta \mathrm{Ct}}$ method [14]. The primer sequences were as follows: Lipin-1 forward TATGACACG GCTTGTTCC, reverse GTGGCTGCCCTGTATTTC; $\beta$ actin forward CCTAGACTTCGAGCAAGAGA, reverse GGAAGGAAGGCTGGAAGA (Generay, Shanghai, China).

2.8. Western Blot. Rats were euthanized while under anesthesia, and hippocampal CA1 tissues were immediately removed and stored at $-80^{\circ} \mathrm{C}$ until processed for protein extraction. Cellular and the tissue proteins were extracted on ice using cold RIPA buffer (Beyotime, Shanghai, China) supplemented with a protease inhibitor mixture and phenylmethylsulfonyl fluoride (Beyotime, Shanghai, China). The samples were subjected to three episodes of ultrasound for $10 \mathrm{~s}$ each, if necessary, and then centrifuged at $12,000 \mathrm{rpm}$ for $20 \mathrm{~min}$ at $4^{\circ} \mathrm{C}$. The supernatant was heated at $95^{\circ} \mathrm{C}$ for $5 \mathrm{~min}$ and retained for Western blot analyses. Total protein concentrations were determined using the BCA kit (Beyotime, Shanghai, China). Equal amounts of proteins were fractionated using $8 \%$ or $12 \%$ sodium dodecyl sulfate polyacrylamide gel electrophoresis and transferred onto PVDF membranes. The membranes were blocked with $5 \%$ bovine serum albumin (BSA) for $1 \mathrm{~h}$ at room temperature and incubated overnight at $4^{\circ} \mathrm{C}$ with the primary antibodies, anti-Lipin1 (1:250, ab181389), antitubulin ( $1: 5,000$, abway), anti-glyceraldehyde-3-phosphate dehydrogenase (GAPDH; 1:5,000, proteintech), anti-Ppkd (1:500, Ser744/748, CST2054), anti-Pssh1 (1:250, pSer978, Novus, NBP1-50636), anti-Limkinase1 (1:500, phosphoT508, ab38508), anti-Pcofilin (1:250, phosphoS3, ab12866), and anti-Synaptophysin (1:7,500, ab32127). The membranes were then washed three times with TBST for 5 min each and incubated with the secondary antibodies conjugated with HRP $(1: 5000)$ for $1 \mathrm{~h}$ at room temperature followed by 3 washings with TBST for $10 \mathrm{~min}$ each. Detection was performed using an enhanced chemiluminescence detection kit (Merck Millipore, Billerica, MA, USA). Intensities of the blots were quantified using Image J software.

2.9. Transmission Electron Microscopy. The hippocampal CA1 region $\left(1 \mathrm{~mm}^{3}\right)$ was collected, fixed with $2 \%$ glutaraldehyde, dehydrated with use of an ethanol gradient, embedded, and sectioned. Samples were then stained with uranyl acetate followed by lead citrate on the copper grids. Neurons within these hippocampal CA1 regions were then observed 
under a transmission electron microscope (Philips Tecnai 20 U-Twin, Holland).

2.10. Golgi Stain. Brains were impregnated using the Golgi stain kit (Servicebio, Wuhan, China) in accordance with the manufacturers' instructions. Coronal sections $(100 \mu \mathrm{m}$ thickness) from the CA1 hippocampal region were analyzed from microscopic images as processed using Fiji software.

2.11. Enzyme-Linked Immunosorbent Assay (ELISA). Diacylglycerol concentrations within the hippocampal CA1 region were detected using ELISA kits (J\&L Biological, China) with reference to the supplier's description.

2.12. Statistical Analysis. All data are presented as means \pm SEMs and were analyzed using the IBM SPSS Statistics 22.0 program. Comparisons between groups were performed using unpaired $t$-tests, while comparisons involving multiple groups were analyzed using one-way ANOVA. A $P$ value $<$ 0.05 was required for results to be considered as statistically significant.

\section{Results}

3.1. Lipin1 Enhances Viability in PC12 Cells Exposed to High Glucose Concentrations. PC12 cells were cultured for either 24 or $48 \mathrm{~h}$ under different concentrations of glucose and mannitol (25-150 mM, with $25 \mathrm{mM}$ serving as the control concentration), and cell viability was assessed using the CCK-8 assay. As shown in Figure 1(a), there was a concentration-dependent decrease in cell viability in response to these treatments. Cells cultured with glucose and mannitol concentrations of $150 \mathrm{mM}$ for 24 or $48 \mathrm{~h}$ and $125 \mathrm{mM}$ for $48 \mathrm{~h}$ showed statistically significant decreases in viability. Cell viability is decreased when cultured with glucose in $100 \mathrm{mM} / 48 \mathrm{~h}$ compared to mannitol in the same conditions $(P<0.05)$. To eliminate potential cytotoxic effects of increased osmolarity, the 125 and $150 \mathrm{mM}$ glucose concentrations were excluded from further analyses. As decreases in cell viability with $100 \mathrm{mM} / 48 \mathrm{~h}(P<0.01)$ were more evident than that obtained with $75 \mathrm{mM} / 48 \mathrm{~h}$ $(P<0.05)$, the $100 \mathrm{mM} / 48 \mathrm{~h}$ condition was chosen for use as the high glucose $(\mathrm{HG})$ condition in subsequent experiments.

The mRNA $(P<0.01)$ and protein $(P<0.01)$ levels of Lipin1 were significantly decreased in the HG condition (Figure 1(b)). Cultured with mannitol, the mRNA and protein levels of Lipin 1 were no statistic difference at the condition of $100 \mathrm{~mm} / 48 \mathrm{~h}$ compared to control (Figure $1(\mathrm{c})$ ). Therefore, given these results indicating that Lipin1 was associated with these reductions in cell viability from $\mathrm{HG}$, a LV for Lipin 1 upregulation and downregulation was constructed for use in transfection within these PC12 cells to further clarify this relationship between Lipin1 and cell viability. Infection efficiency was visualized using fluorescence microscopy, with the result that $>90 \%$ of the cells were infected. This infection efficiency was further verified using $\mathrm{qPCR}$ and Western blot analysis (Figure 1(d)). Levels of Lipin1 mRNA in the LV-Lipin1 group were significantly increased as compared with those in the LV-Lipin1-Con group $(P<0.001)$, whereas mRNA levels of Lipin1 in the LV-
Lipin1ShRNA group were significantly decreased as compared with those in the LV-Lipin1ShRNA-Con group $(P<0.05)$. Similar changes in Lipin 1 protein levels as that observed for mRNA were obtained in these conditions.

Cells infected with Lv-Lipin1-Con and Lv-Lipin1 were subjected to $\mathrm{HG}$ conditions ( $\mathrm{HG}+\mathrm{Lv}-\mathrm{Con}$ and $\mathrm{HG}+\mathrm{Lv}-$ Lipin1 groups) to assess the role of Lipin1 in glucoseinduced injury. Cells infected with Lv-Lipin1ShRNA-Con and Lv-Lipin1ShRNA were treated with the (control) $25 \mathrm{mM}$ glucose and mannitol concentration (NG+Lv-Con and NG+Lv-Lipin1ShRNA group) (Figure 1(e)). Cell viability within the $\mathrm{HG}+\mathrm{Lv}$-Lipin1 group was significantly increased as compared with that obtained in the HG+LvCon group $(P<0.01)$, while cell viability within the NG + Lv-Lipin1ShRNA group was significantly decreased as compared with that of the NG+Lv-Con group $(P<0.001)$. Lipin1 overexpression attenuated the HG-induced decrease in the viability of PC12 cells, while the silencing of Lipin 1 led to a decrease in the viability of PC12 cells as observed under NG condition. These results suggest that Lipin1 may be related to the changes in PC12 cell viability as observed in response to $\mathrm{HG}$ exposure.

3.2. Lipin1 Is Reduced in the Hippocampal CA1 Region of Diabetic Rats. From the third day after STZ injection, body weights were significantly decreased $(P<0.001)$, and FBG levels significantly increased $(P<0.001)$ in this rat model of diabetes as compared with that of the wild-type (WT) group (Figure 2(a)). These results suggest that a diabetic condition was successfully achieved in these rats. Associated with this diabetic condition were significant decreases in mRNA $(P<0.01)$ and protein $(P<0.05)$ levels of Lipin1 within the hippocampal CA1 region in these diabetic rats as compared with controls (Figure 2(b)).

3.3. Lipin1 Content within the CA1 Region Affects Cognitive Functions in Rats. Within the diabetic rats, those showing $\mathrm{DE}$ as revealed by their behavioral test performance were selected to assess whether Lipin1 was related to cognitive function, as well as to determine whether Lipin1 content within the CA1 region was changed in response to a stereotactic injection of the virus into this site. Two complementary approaches to examine this issue involved LV-Lipin1ShRNA injection into WT rats to reduce Lipin1 and LV-Lipin1 injection into DE rats to increase Lipin1. The blank vector was injected as a control (Figure 3(a)). Infection efficiencies were estimated with the use of qPCR and Western blot analysis (Figure 3(b)). The mRNA levels of Lipin1 in the WT+LVLipin1ShRNA $(P<0.05)$ and DE+LV-Con $(P<0.01)$ groups were significantly decreased as compared with those in the WT+LV-Con group, whereas these Lipin1 mRNA levels in the DE+LV-Lipin1 group were significantly increased as compared with those in the DE+LV-Con group $(P<0.01)$. Similar changes in Lipin1 protein levels were observed in these conditions.

Behavioral tests were performed at 14 days after stereotactic injection of the virus to assess the effects of the changes in Lipin1. In the open field test, no differences were obtained in the number of crossings of the squares among the groups 


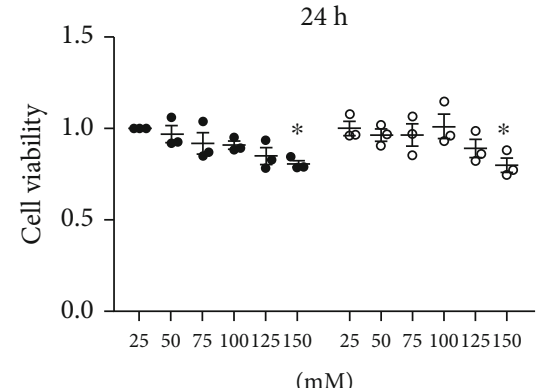

- Glucose

- Mannitol
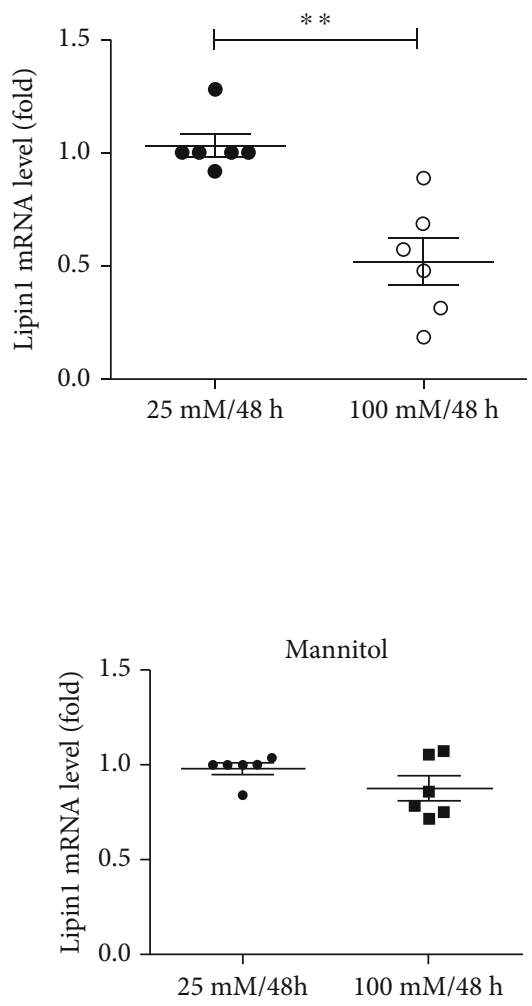

LV-Lipin1-Con

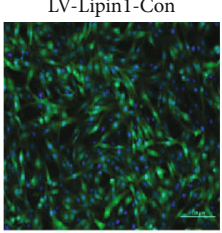

LV-Lipin1 ShRNA-Con
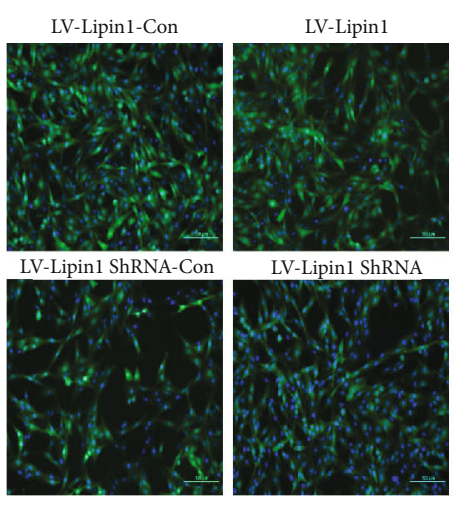
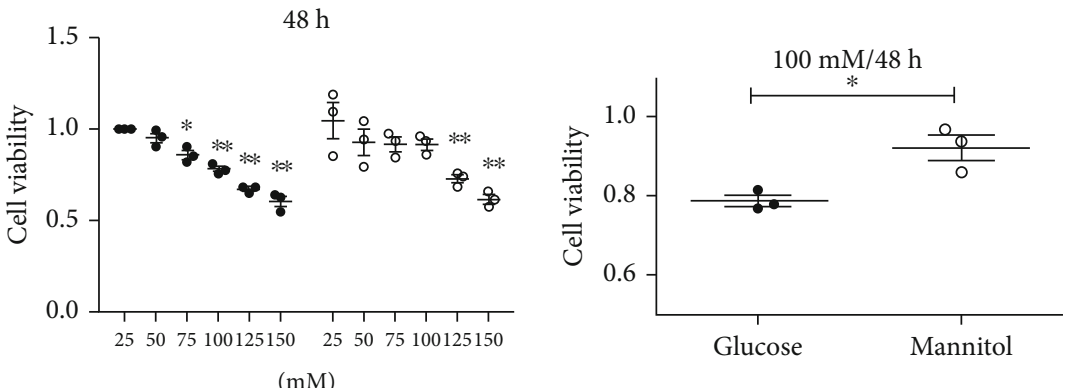

(a)

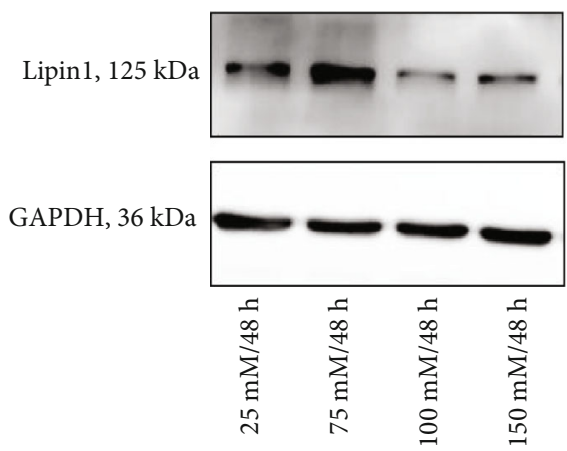

(b)
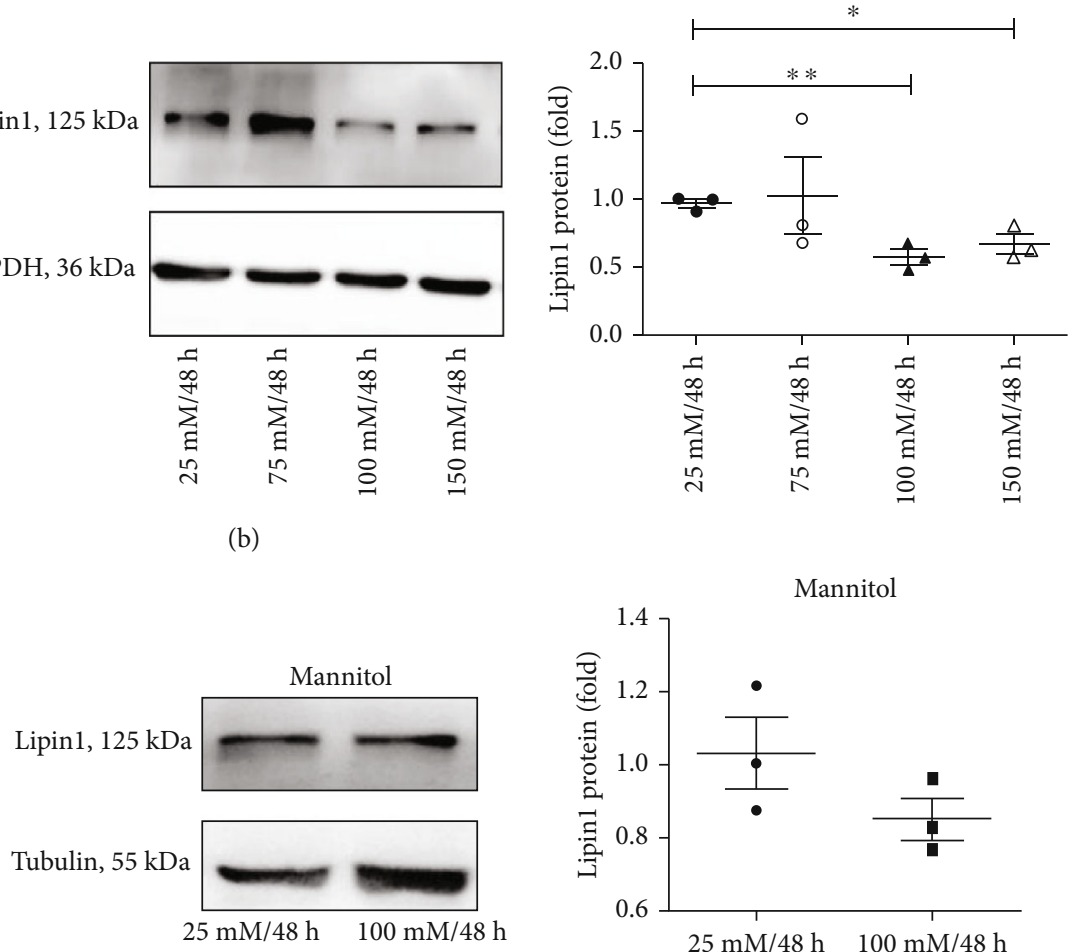

(c)

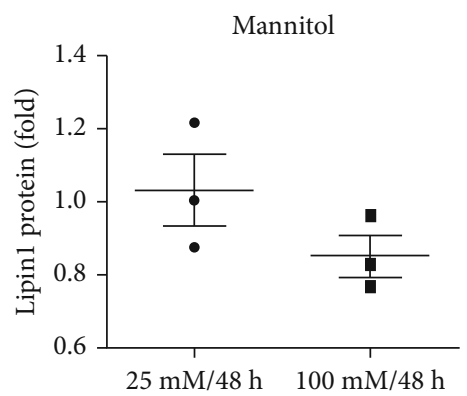

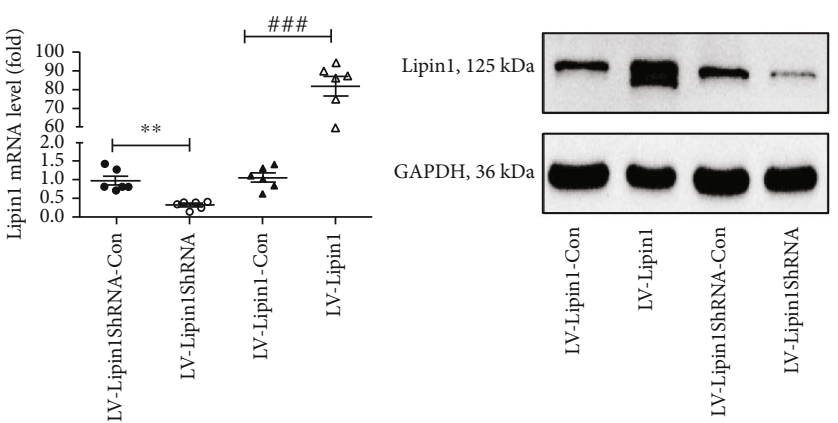

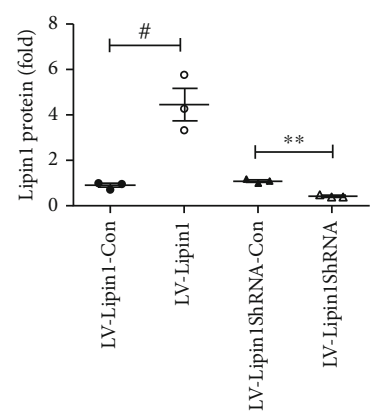

(d)

Figure 1: Continued. 


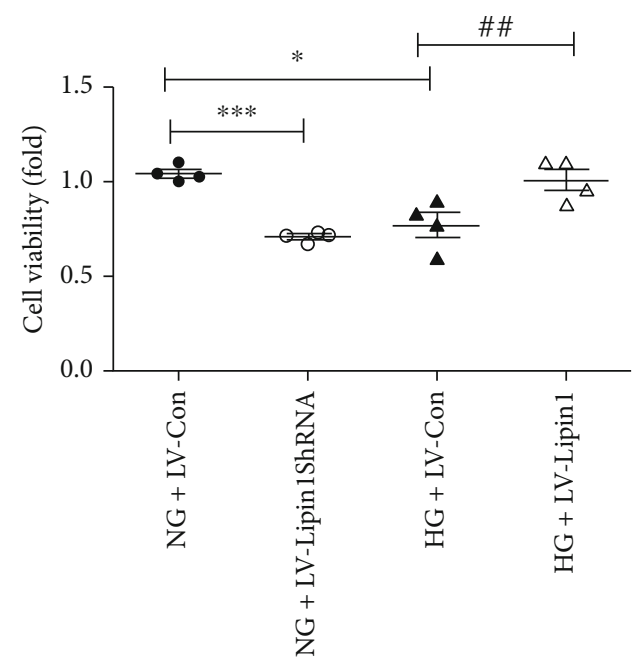

(e)

FIGURE 1: Changes in PC12 cell viability in response to high glucose toxicity are related to Lipin1. (a) Cell viability after 24 or $48 \mathrm{~h}$ of treatment with different concentrations of glucose and mannitol, and the cells were incubated in the same concentrations (100 mM) of glucose and mannitol for $48 \mathrm{~h}$. The cell viability of each group was standardized to that of the control group. (b) Incubated with glucose, lipin $1 \mathrm{mRNA}$ and protein expressions as determined using qPCR and Western blot. (c) Incubated with mannitol, lipin1 mRNA and protein expressions as determined using qPCR and Western blot. ${ }^{*} P<0.05,{ }^{*}{ }^{*} P<0.01$, versus $25 \mathrm{mM} / 48 \mathrm{~h}$. (d) Cells infected with Lv-Lipin1, Lv-Lipin1-Con, Lv-Lipin1ShRNA, and Lv-Lipin1ShRNA-Con for three days. Nucleus were stained blue with 4',6-diamidine-2-phenylidole dihydrochloride (DAPI). Infection efficiencies were determined using fluorescence microscopy while postinfection Lipin1 mRNA and protein expressions were determined using qPCR and Western blot analysis. ${ }^{*} P<0.05,{ }^{* *} P<0.01$, versus LV-Lipin 1 ShRNA-Con group;

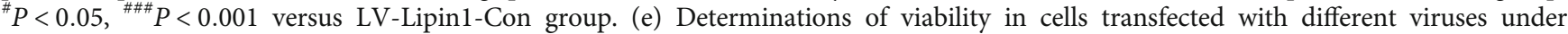
different treatment conditions. ${ }^{*} P<0.05,{ }^{* * *} P<0.001$ versus NG+LV-Con group; ${ }^{\# \#} P<0.01$, versus HG+LV-Con goup. Scale bar in $(\mathrm{d})=$ $100 \mu \mathrm{m}$. Data are expressed as means \pm SEMs. Results are representative of three independent experiments.

$(P>0.05$, Figure 3(c)), indicating that this stereotactic injection of the virus did not affect the general locomotor activity of these rats. Interestingly, the DE+LV-Con $(P<0.01)$ and WT+LV-Lipin1ShRNA $(P<0.05)$ groups spent less time in the central area than the WT+LV-Con group. In contrast, the DE+LV-Lipin1 group spent significantly more time in the central area than the $\mathrm{DE}+\mathrm{LV}$-Con group $(P<0.05)$. Taken together, these results indicate that a reduction in hippocampal CA1 Lipin1 was associated less exploratory activity, suggesting that higher levels of anxiety may be present in these rats.

Results of the NOR test are presented in Figure 3(d). While there were no differences in total exploration times directed to the objects among the four groups, significant differences were present with regard to exploration of the novel versus familiar objects. The WT+LV-Lipin1ShRNA $(P<0.05)$ and DE+LV-Con $(P<0.01)$ groups showed significantly lower DI scores than that of the WT+LV-Con group. In the DE+LV-Lipin1 group, DI scores were significantly greater than that of the $\mathrm{DE}+\mathrm{LV}-\mathrm{Con}$ group $(P<0.05)$. These results indicate that Lipin 1 reversed the STZ-induced learning and memory impairments present in DE rats.

In the first two days of the MWM test, no differences were observed among the four groups (Figure 3(e)). On the third and the fourth day, escape latencies of the DE+LVCon group were significantly increased as compared with those of the WT+LV-Con group $(P<0.01)$. Significant increases in escape latency were also observed in the
$\mathrm{DE}+\mathrm{LV}$-Con versus the DE+LV-Lipin1 groups on the fourth day $(P<0.01)$. Although escape latencies of the WT+LVLipin1ShRNA group were greater than that of the WT+LVCon group, these differences failed to achieve statistical significance $(P>0.05)$. With regard to the number of platform crossings in the spatial exploration test, the DE+LV-Con and WT+LV-Lipin1ShRNA groups showed significantly fewer crossings as compared with that in the WT+LV-Con group $(P<0.05)$. The number of platform crossings in the DE+LV-Lipin1 group was significantly increased as compared with that of the DE+LV-Con group $(P<0.01)$. These results demonstrate that Lipin1 facilitated the performance of these rats in the MWM test, suggesting that Lipin1 reversed the cognitive deficits observed in these diabetic rats.

3.4. Lipin1 within the CA1 Region Affects the Morphological Structure of Dendritic Spines and Synapses. Electron microscopy and Golgi staining were used to observe the changes in the neurons within the hippocampal CA1 region of rats. As shown in Figure 4(a), the number of synapses per visual field in the WT+LV-Lipin1ShRNA and the DE+LV-Con groups were significantly less than that in the WT+LV-Con group $(P<0.001)$. Significantly, more synapses were found in the DE+LV-Lipin1 versus DE+LV-Con group $(P<0.01)$. The spine densities, as shown in Figure 4(b), reveal that a significant reduction was present in the $\mathrm{WT}+\mathrm{LV}$-Lipin 1 ShRNA $(P<0.001)$ and the DE+LV-Con $(P<0.001)$ groups as 

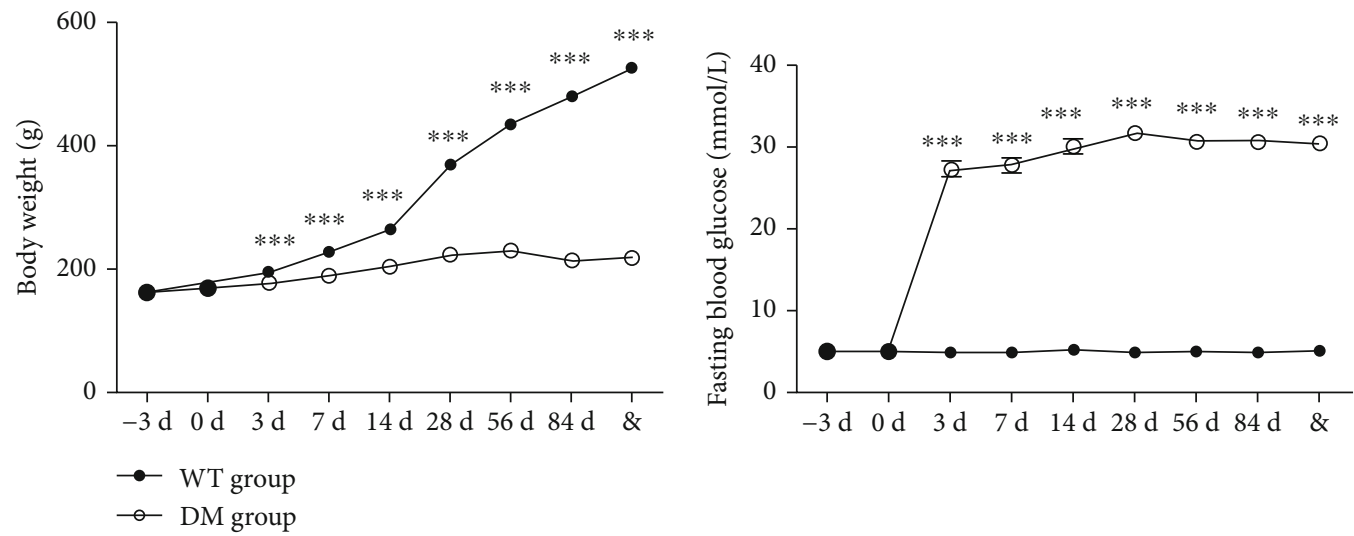

(a)
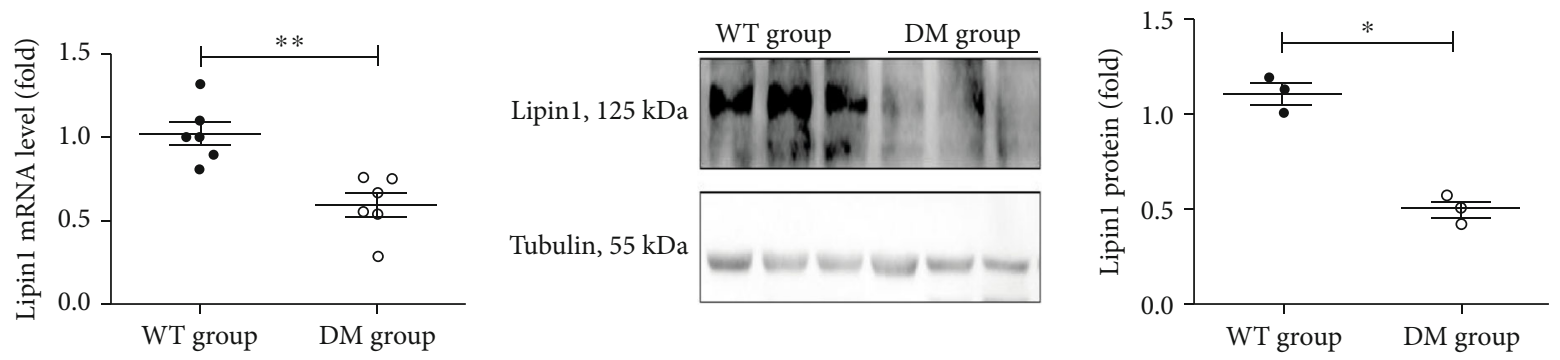

(b)

FIGURE 2: Changes in Lipin1 within the hippocampal CA1 region of diabetic rats. (a) Changes in body weights and blood glucose levels in rats after STZ injection, indicating the induction of a diabetic condition ( $n=30$ animals/group). (b) Expression of Lipin1 levels in the hippocampal CA1 region as determined using qPCR ( $n=6$ animals/group) and Western blot analysis ( $n=3$ animals/group). Data are expressed as the means $\pm \mathrm{SEMs},{ }^{*} P<0.05,{ }^{* * *} P<0.01,{ }^{* * *} P<0.001$ versus wild type group and \& means as determined at 14 days after STZ injection.

compared with that observed in the WT+LV-Con group. Spine densities in the DE+LV-Lipin1 group were significantly greater than that in the DE+LV-Con group $(P<0.001)$.

\subsection{Lipin1 Regulates Activity of the PKD/Limk1/Cofilin} Signaling Pathway. Signaling molecule downstream of Lipin1 was detected to further explore the possible neuronal mechanisms of Lipin1 (Figure 5). Levels of DAG in the WT+LV-Lipin1ShRNA and the DE+LV-Con groups were significantly decreased as compared with those in the WT+LV-Con group $(P<0.001)$, whereas DAG levels in the DE+LV-Lipin1 group were significantly increased as compared with those in the DE+LV-Con group $(P<0.001$, Figure 5(a)). These results are consistent with the changes observed in Lipin1 after virus injection. Signaling pathway proteins of P-Limk1/P-Cofilin were determined using the Western blot assay (Figure 5(b)). As compared with that observed in the WT+LV-Con group, the WT+LVLipin1ShRNA and the DE+LV-Con groups showed substantial reductions in P-PKD, P-limk1, P-cofilin, and Syn protein expression levels, whereas these protein levels were significantly increased in the DE+LV-Lipin1 group as compared with those in the DE+LV-Con group. In contrast, protein levels of P-ssh1 in the WT+LV-Lipin1ShRNA and $\mathrm{DE}+\mathrm{LV}$-Con groups were increased as compared with the WT+LV-Con group $(P<0.05)$, but were decreased in the DE+LV-Lipin1 as compared with that of the DE+LVCon group $(P<0.05)$. These results demonstrate that the regulation of these proteins is clearly influenced by Lipin1.

\section{Discussion}

The neurological complications associated with diabetes have attracted considerable attention of late due to their potential in complicating the pathogenesis and therapeutic effects in this condition. Research on the mechanisms of DE have mainly focused on axonal and dendritic changes [15], the prolonged severity associated with deficiencies in insulin [16], abnormal insulin signaling [17, 18], oxidative stress, advanced glycation end-products [19], and synaptic plasticity [20]. Previous results from our laboratory have indicated that increases in Lipin1 content are associated with improvements in nerve conduction velocity and relief of painful neuropathy in DM rats [8]. In the muscle, decreased lipin1 levels and statin treatment staggered to induce autophagy by reducing mTOR, and the ability of autophagy flux will be reduced due to increased PA and decreased activation of PKD, which further leads to impaired autophagy clearance [6]. Moreover, we observed significant impairments in cognitive functions in Lpin $1^{\text {fld } / J ~ m i c e ~[9] . ~ T h e s e ~ r e s u l t s ~ s u g g e s t ~ t h a t ~ D P N ~ m a y ~}$ be related to a decline in Lipin1. In the present study, both in vitro and in vivo models were employed in an attempt to 


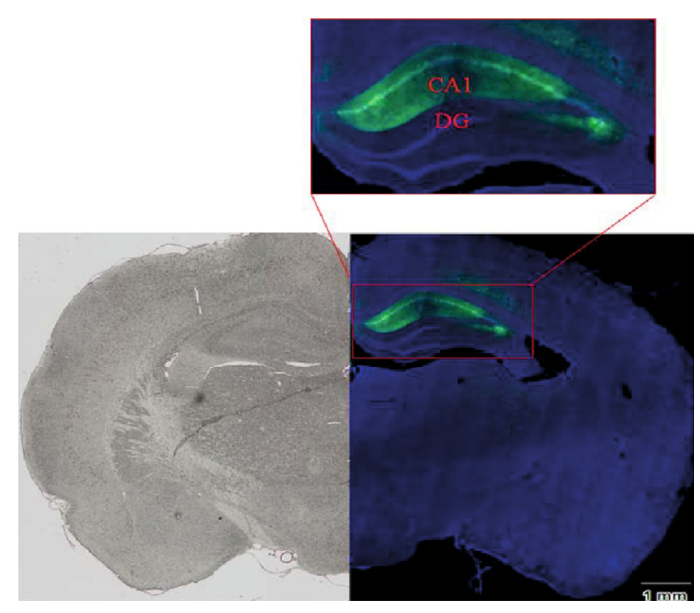

(a)
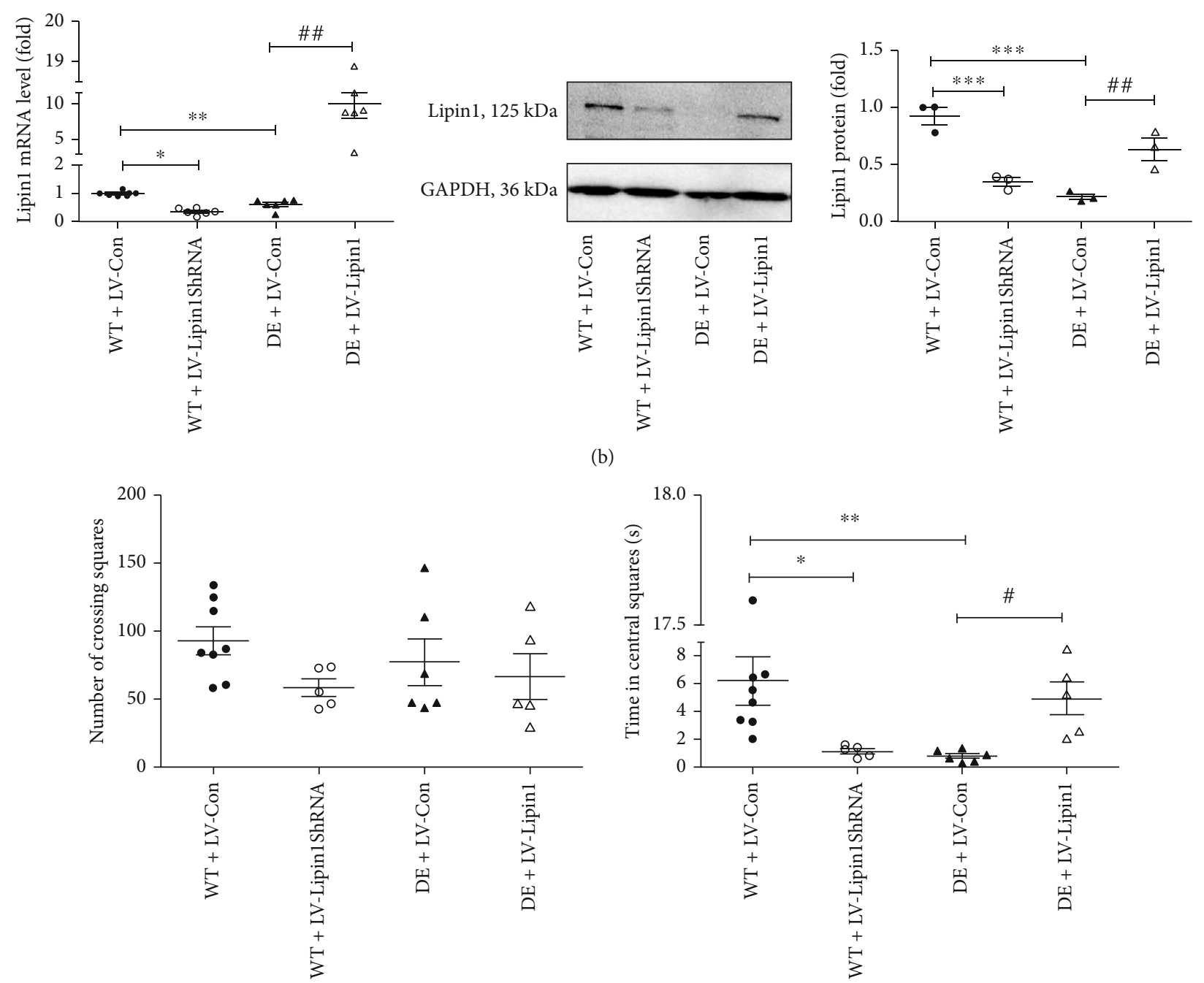

(b)

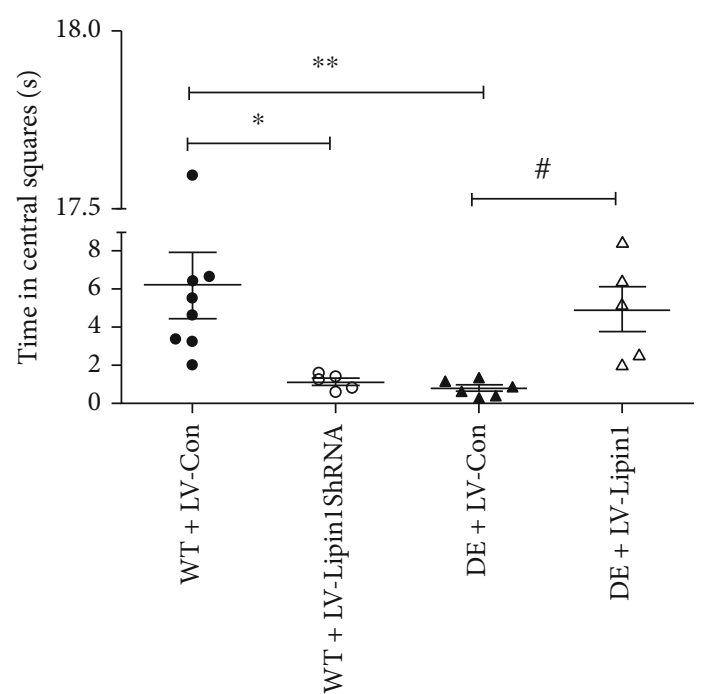

(c)

Figure 3: Continued. 

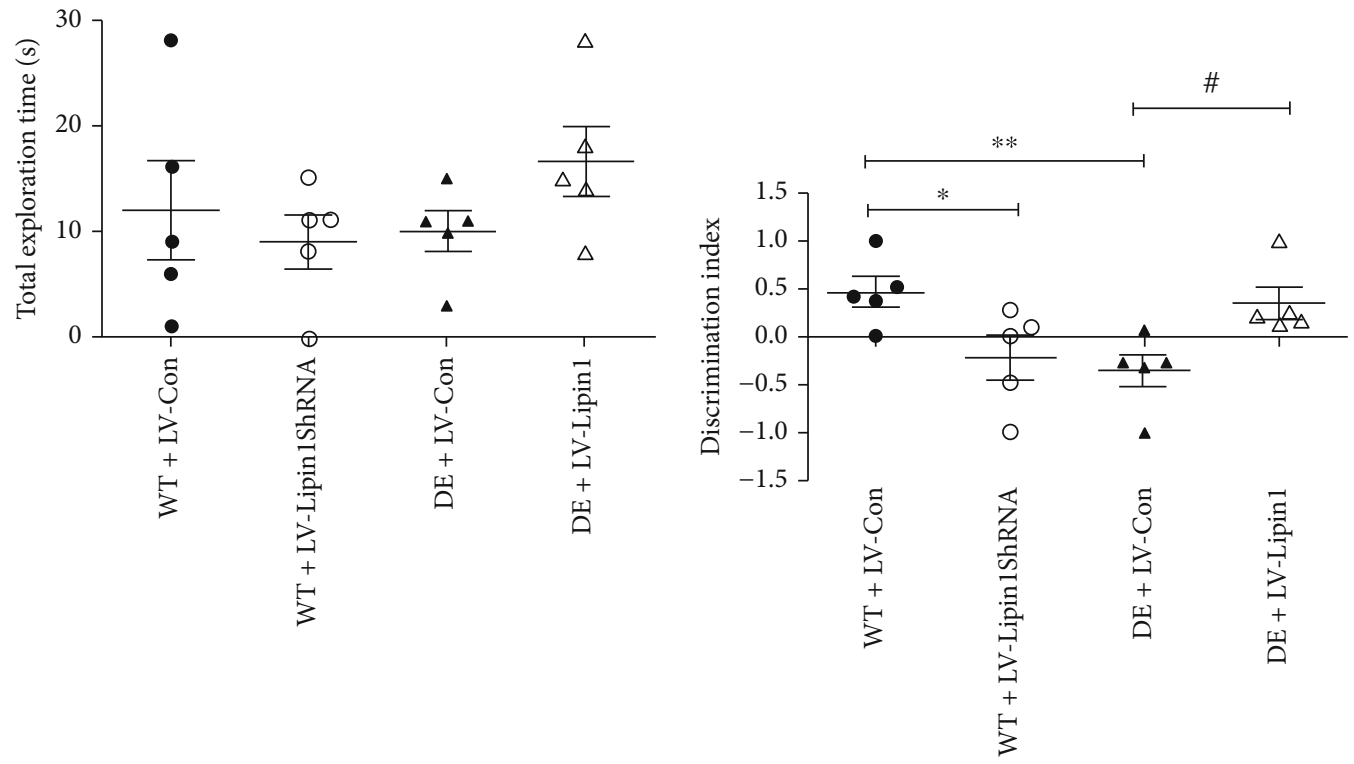

(d)
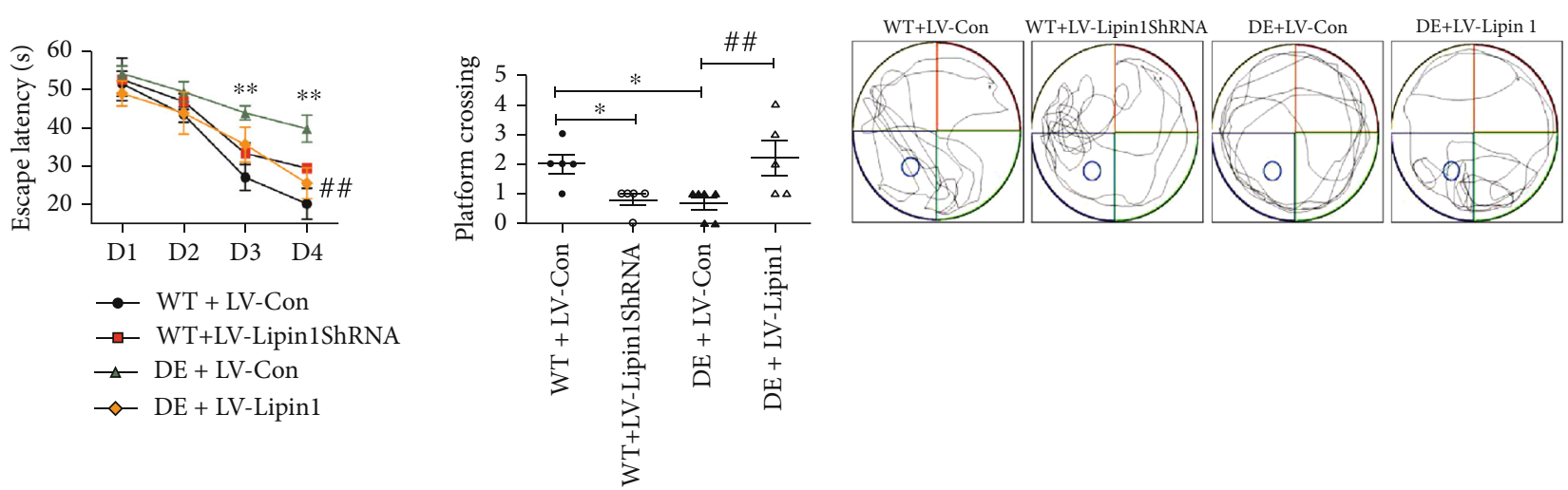

(e)

FIGURE 3: Results of behavioral tests in rats in response to LV injection. (a) Illustration of the viral infusion of LV-Lipin1. (b) The Lipin1 content in the CA1 region after LV injection as determined with use of qPCR and Western blot analysis. (c) Open field test. The number of squares crossed indicates general locomotor activity while time in central area indicates the amount of exploration. (d) Novel object recognition test. The total exploration time and discrimination index were analyzed. (e) Morris water maze test. The escape latencies on different training days and the number of platform crossings were determined. Movements of the rats were also recorded using tracking software. Data represent the means \pm SEMs, $n=5-8$ animals per group. ${ }^{*} P<0.05,{ }^{* *} P<0.01,{ }^{* * *} P<0.001$ versus WT+LV-Con group; ${ }^{\#} P<0.05,{ }^{\# \#} P<0.01$ versus DE+LV-Con group. Scale bar in $(\mathrm{a})=1 \mathrm{~mm}$.

examine the role and some of the mechanisms of Lipin1 in the regulation of cognitive disorders in $\mathrm{DE}$ rats.

PC12 cell, a mature cell line from a rat pheochromocytoma, has been widely used in neurophysiological studies. In particular, these cells have served as a commonly used cellular model for studying the mechanisms underlying glucose neurotoxicity [21-23]. With regard to Lipin 1, results from in vitro experiments which have shown that Lipin1 concentrations were reduced when PC12 cells were treated with HG and Lipin1 alleviated HG-induced neurotoxicity in these cells. In an attempt to expand upon these findings, we constructed a model of T1DM in rats as achieved with an intraperitoneal injection of STZ as a means to assess these effects of Lipin1 in vivo.

Based upon the results obtained with this in vivo model, it is clear that Lipin1 affects cognitive functions in rats. The approach used in these experiments involved injections of Lipin1 LVs into the hippocampal CA1 region of these rats. At 14 days after injection, this LV can effectively integrate the transgene and remain expressed for an extended period of time [24]. Following this treatment, the rats were tested in a series of behavioral paradigms including, open field, NOR, and MWM, to assess their activity/anxiety and cognitive functions. The open field test provides a means to assess spontaneous activity, investigation, and anxiety. With this test, animals showing anxiety and depression in this novel environment increase their autonomous activity while they decrease their exploratory activity [25-27]. While the overall general activity of the rats was not different among the groups in this test, the DE group treated with LV-Lipin in the CA1 region showed increased amounts of time spent in the central squares as compared with that observed in DE 

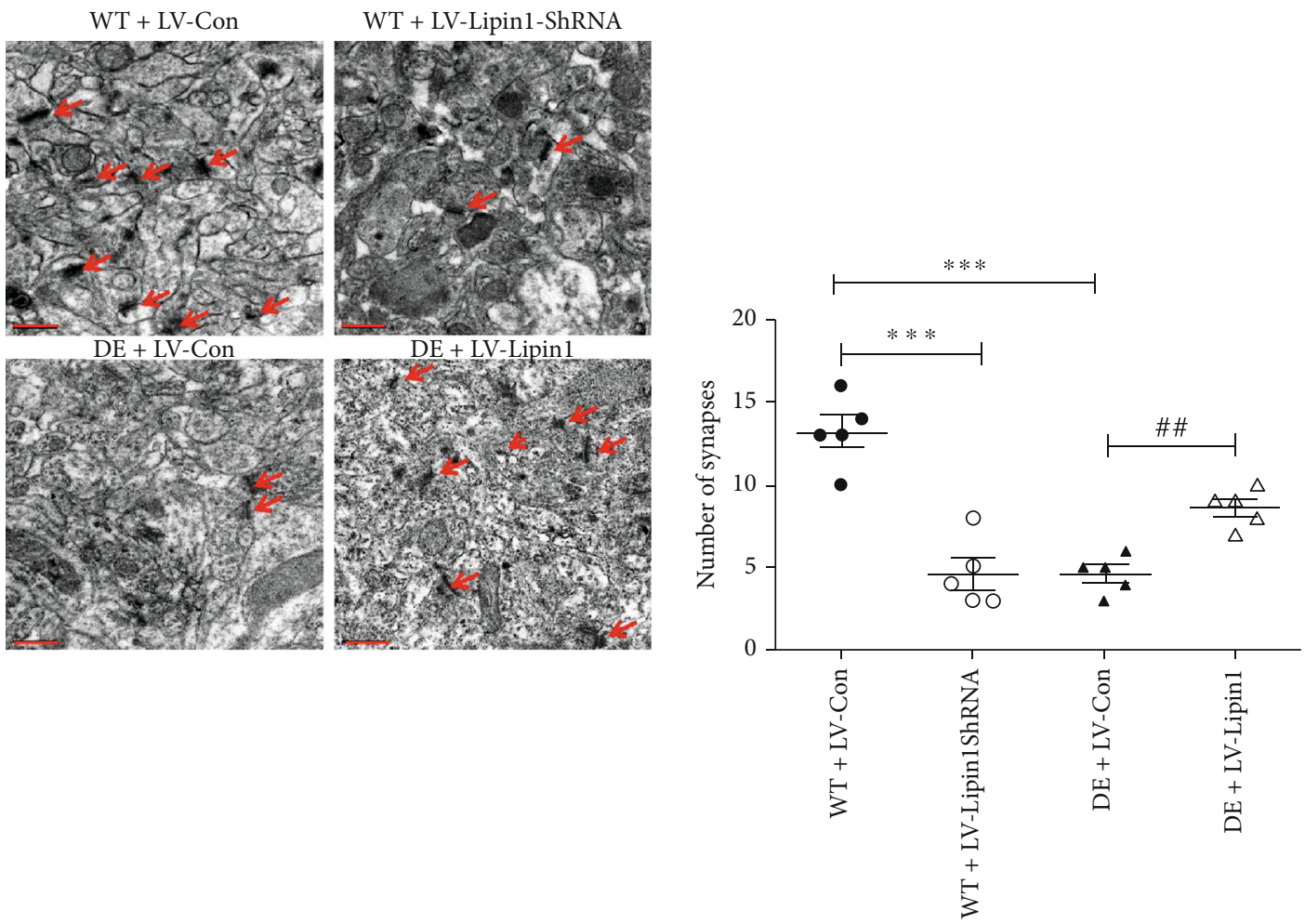

(a)

WT + LV-Con

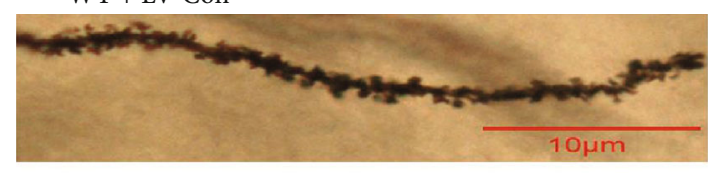

WT + LV-Lipin1ShRNA

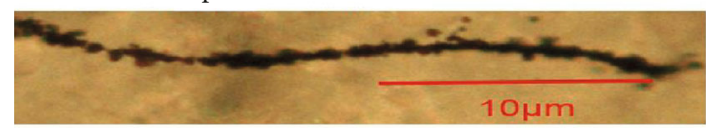

$\mathrm{DE}+\mathrm{LV}-\mathrm{Con}$

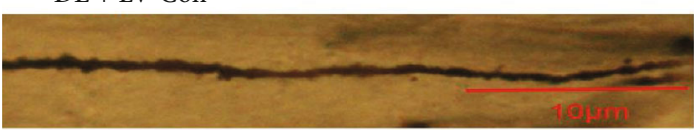

$\mathrm{DE}+\mathrm{LV}$-Lipin 1

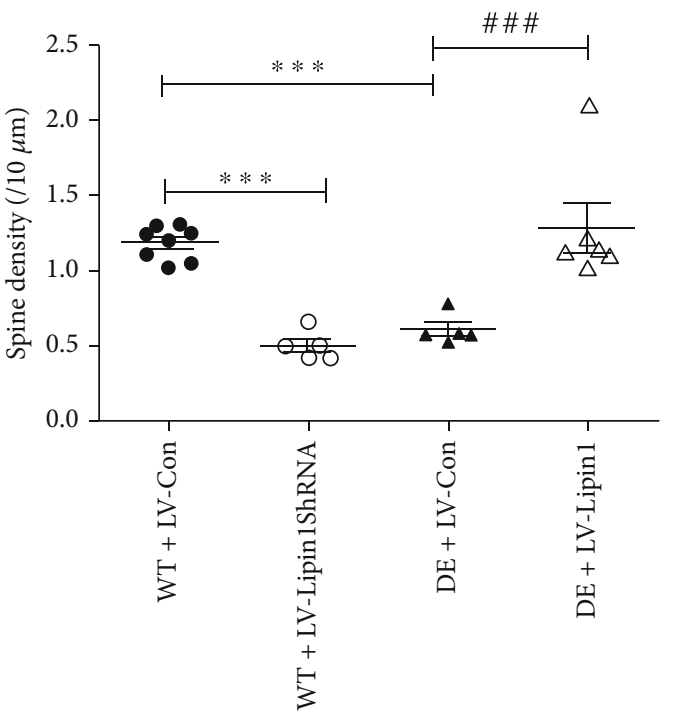

(b)

Figure 4: Changes in the morphological structure of dendritic spines and synapses in response to LV injection. (a) Synapses were observed under transmission electron microscopy with arrow indicating location of synapses (scale bar $=0.5 \mu \mathrm{m}$ ). Arrows indicate synapses. (b) Representative Golgi-stained dendritic spine image from the hippocampal CA1 region (scale bar $=10 \mu \mathrm{m}$ ). The images presented were screenshots. The source of these screenshots was shown in the supplementary material Figure S1. Data represent the means \pm SEMs, $n=5-8$ animals per group. ${ }^{* * *} P<0.001$ versus $W T+L V-C o n$ group; ${ }^{\# \#} P<0.01$, ${ }^{\# \#} P<0.001$ versus DE+LV-Con group. 


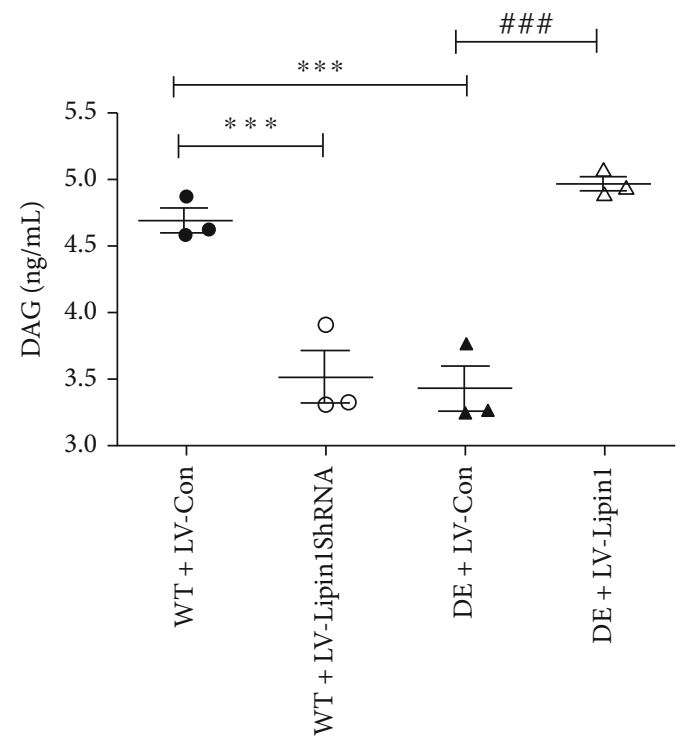

(a)
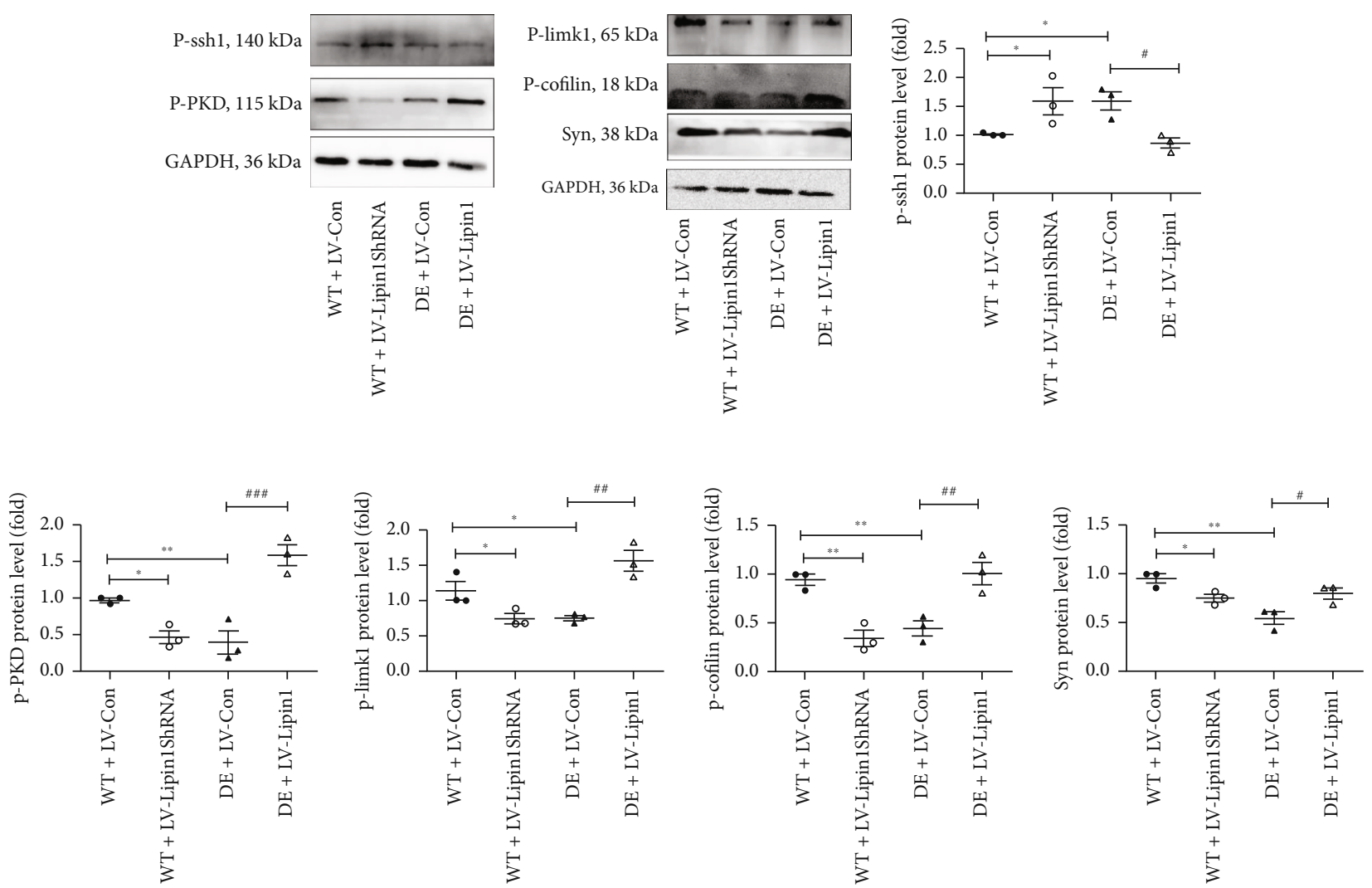

(b)

FIGURE 5: Effect of Lipin1 on activation of DAG, p-ssh1, p-PKD, P-limk1, P-cofilin, and Syn. (a) Levels of DAG were determined using the ELISA kit. (b) Representative Western blot gel results and summary of data showing expression levels of the PKD/Limk1/Cofilin signaling pathway proteins in the CA1 region. ${ }^{*} P<0.05,{ }^{* *} P<0.01$, versus $\mathrm{WT}+\mathrm{LV}$-Con group; ${ }^{\#} P<0.05,{ }^{\# \#} P<0.01$, versus $\mathrm{DE}+\mathrm{LV}-\mathrm{Con}$ group. Results are representative of at least three independent experiments.

rats receiving a LV-control injection. When the expression of Lipin1 was decreased in the CA1 region of the WT group, the time spent in the central squares decreased. Accordingly, Lipin1 enhanced the exploration of DE rats, whereas the absence of Lipin1 was associated with a decrease in this exploratory activity. The decreased exploration of rats in the DE and WT+LV-Lipin1ShRNA groups also reflected an increased degree of anxiety in these two groups. The NOR test is based on the nonspatial short-term memory of animals' spontaneous preference for novel objects [28-30]. In 
the testing period phase of the NOR test, neither hyperglycemia nor the stereotactic injection treatments altered the total amount of time spent investigating the objects, but salient differences were observed among the different treatment groups with regard to their preferences for investigating the familiar versus novel objects. Specifically, we found that Lipin 1 treatment prevented deficits produced by hyperglycemia in this test of discriminative memory. Finally, we used the MWM as a means to assess visually related spatial and working memory $[13,31]$. Notably, compared with the WT+LV-Con group, the escape latency of WT+LVLipin1ShRNA group did not differ. We analyzed the possibility that after the open field and the NOR tests, the rats may have a certain learning ability to compensate. The spatial exploration test results suggested that Lipin1 can improve the spatial learning ability in DE rats. The reduced Lipin 1 expression in the CA1 region of WT rats may cause impairment in the spatial learning ability. When collating the results from these behavioral tests, it seems clear that Lipin 1 plays a critical role in the behavioral responses examined. In specific, Lipin1 improves cognitive functions and reduces anxiety in DE rats, whereas a reduction in Lipin1 content within the hippocampal CA1 region of WT rats impairs cognitive functions and increases anxiety in these rats.

Next, we examined the effects of these treatments on neuronal structures within the CA1 region with the use of electron microscopy and Golgi staining. We found that the changes in the number of synapses and densities of dendritic spines in each group of rats were consistent with the changes observed in Lipin 1 contents and cognitive responses in these rats. That is, perturbations of these neuronal structures were associated with deficits in cognitive functions. Interestingly, in sleep-deprived mice, the number of dendritic spines within the hippocampal CA1 region was selectively reduced, effect which was accompanied with increases in activity of the filamentous actin-severing protein, cofilin [32]. Cofilin, an actin-binding protein commonly found in the cytoskeletal proteins of eukaryotes, promotes cell migration and movement. Activation of cofilin increases f-actin depolymerization and disassembly, causing atrophy and loss of dendritic spines [33-36]. Cofilin is negatively regulated by phosphorylation, inactivated by the Ser3 of limk1, and activated by the dephosphorylation of ssh1 [37, 38]. Through the phosphorylation and inactivation of the SSH at Ser-978, active PKD isoforms markedly increase the phosphorylation of cofilin at Ser-3 to inhibit the actin-driven directed cell migration [39]. During cancer cell migration, SSH1 is identified as a substrate for PKD1-regulation of cofilin activation [40]. With regard to our current results, the Lipin1-related neuronal and cognitive changes within the CA1 region we observed here are similar to that obtained in response to that of cofilin. In addition, we also detected the synthesis of DAG and alterations in the expressions of DAG, p-PKD, p-ssh1, p-limk1, and p-cofilin in these rats. Our findings of decreased levels of DAG and $\mathrm{p}-\mathrm{PKD}$ within the $\mathrm{DE}+\mathrm{LV}-\mathrm{Con}$ and the $\mathrm{WT}+\mathrm{LV}-$ Lipin1ShRNA groups were in accord with results from previous reports $[6,9,41,42]$. Interestingly, as shown in hepatocytes, DAG can produce insulin resistance and con- tribute to the promotion of type 2 diabetes. As PKD is also involved as an effector of DAG, a deficiency of PKD1 in adipocytes has the effect of increasing insulin sensitivity [43-45]. It has also been reported that stress-induced nascent granule degradation (SINGD) is involved in the development of diabetes as a result of damage to pancreatic beta cells. As PKD is a negative regulator of SINGD, an inhibition of PKD would exacerbate SINGD, thereby contributing to an acceleration of diabetes [46]. These likely differential central and peripheral mechanisms involving complex actions of Lipin1, DAG, and PKD will require further in-depth study. Moreover, the contents of p-limk1 and p-cofilin were decreased in the DE+LV-Con and the WT+LV-Lipin1ShRNA groups, whereas that of p-ssh1 was increased. Accordingly, we also detected changes in synaptophysin, which plays a very important role in regulating activity-dependent synapses [47]. Consistent with the previous results, synaptophysin was decreased in DE rats $[48,49]$. In the DE+LV-Lipin1 rats, we observed an increase in synaptophysin, an effect which was associated with improved cognitive functions. A CA1-specific downregulation of Lipin1 in WT rats can have the effects of enhancing cofilin activity and increasing depolymerization and, in this way, reduce dendritic spines, synaptophysin, and cognitive functions. In contrast, an upregulation of Lipin 1 in the CA1 of DE rats would suppress cofilin activity, thus improving cognitive functions in $\mathrm{DE}$ rats through the PKD/Limk/ Cofilin signaling pathway.

\section{Conclusions}

In summary, our results support the hypothesis that sustained hyperglycemia reduces Lipin1 expression within the hippocampal CA1 region to produce a cognitive dysfunction in rats. Neuroprotection of Lipin 1 may thus serve as an important neuronal mechanism to ameliorate the cognitive impairments in DE animal models. These neuroprotective effects of Lipin1 are associated with inhibition of the $\mathrm{PKD} /$ Limk1/Cofilin signaling pathway. Taken together, these findings suggest that Lipin 1 and/or the PKD/Limk1/Cofilin signaling pathway may represent effective protective targets for the development of novel treatments of DE.

\section{Abbreviations}

BSA: $\quad$ Bovine serum albumin

DAG: Diacylglycerol

DAPI: $\quad 4^{\prime}$,6-diamidine-2-phenylidole dihydrochloride

DE: Diabetic encephalopathy

DI: Discrimination index

DM: $\quad$ Diabetes mellitus

DMEM: Dulbecco's modified Eagle's medium

DPN: Diabetic peripheral neuropathy

ELISA: Enzyme-linked immunosorbent assay

FBG: $\quad$ Fasting blood glucose

GAPDH: Glyceraldehyde-3-phosphate dehydrogenase

HG: $\quad$ High glucose

LV: $\quad$ Lentiviral

LVs: $\quad$ Lentiviral vectors 
MWM: $\quad$ Morris water maze

mTOR: Mammalian target of rapamycin

NG: $\quad$ Normal glucose

NOR: $\quad$ Novel object recognition

PA: $\quad$ Phosphatidic acid

PFA: $\quad$ Paraformaldehyde

p-limk1: $\quad$ Protein LIM kinase 1

p-PKD: $\quad$ Phosphorylated protein kinase D

qRT-PCR: Quantitative real-time polymerase chain reaction

ShRNA: Short hairpin RNA

STZ: $\quad$ Streptozocin

SINGD: Stress-induced nascent granule degradation.

\section{Data Availability}

The data used to support the findings of this study are included within the article.

\section{Conflicts of Interest}

The authors declare that there is no conflict of interest regarding the publication of this paper.

\section{Authors' Contributions}

Shihong Chen, Shuyan Yu, and Xianghua Zhuang designed the experiments and made valuable suggestions during the revision of this manuscript. Min Xie, Meijian Wang, and Pan Shang performed the experiments. Min Xie, Wei Liu, and $\mathrm{Min} \mathrm{Xu}$ analyzed the data. Dongqing Jiang, Liping Ju, Fei $\mathrm{Wu}$, and Aili Sun provided technical assistance with the experiments. Min Xie wrote the manuscript. All authors reviewed the manuscript.

\section{Acknowledgments}

This work was supported by the National Natural Science Foundation of China (grant numbers 81670753, 81800722, 81900774), Key Research and Development Plan of Shandong Province (grant number 2018GSF118108), and Natural Science Foundation of Shandong Province (grant number ZR2018PH008).

\section{Supplementary Materials}

Figure S1: the source of the Golgi-stained dendritic spine image screenshots. (Supplementary Materials)

\section{References}

[1] R. N. Dejong, "The nervous system complications of diabetes mellitus, with special reference to cerebrovascular changes," Journal of Nervous and Mental Disease, vol. 111, no. 3, pp. 181-206, 1950.

[2] R. Chen, J. Shi, Q. Yin et al., "Morphological and pathological characteristics of brain in diabetic encephalopathy," Journal of Alzheimer's Disease, vol. 65, no. 1, pp. 15-28, 2018.

[3] S.-Q. Tang, Q.-Y. Jiang, C.-F. Yang, X.-T. Zou, and X.-Y. Dong, "Research and development of Lipin family," Hereditas (Beijing), vol. 32, no. 10, pp. 981-993, 2010.
[4] M. Péterfy, J. Phan, P. Xu, and K. Reue, "Lipodystrophy in the fld mouse results from mutation of a new gene encoding a nuclear protein, lipin," Nature Genetics, vol. 27, no. 1, pp. 121-124, 2001.

[5] M. Valdearcos, E. Esquinas, C. Meana et al., "Subcellular localization and role of Lipin-1 in human macrophages," The Journal of Immunology, vol. 186, no. 10, pp. 6004-6013, 2011.

[6] P. Zhang, M. A. Verity, and K. Reue, "Lipin-1 regulates autophagy clearance and intersects with statin drug effects in skeletal muscle," Cell Metabolism, vol. 20, no. 2, pp. 267-279, 2014.

[7] K. Nadra, A. S. . P. Charles, J. J. Medard et al., "Phosphatidic acid mediates demyelination in Lpin 1 mutant mice," Genes and Development, vol. 22, no. 12, pp. 1647-1661, 2008.

[8] Y. Xu, X. Zhuang, Y. Liu et al., "Over expression of lipin1 attenuates diabetic peripheral neuropathy in rats," Journal of Shandong University (Health Science), vol. 53, no. 9, pp. 5357, 2015.

[9] P. Shang, F. Zheng, F. Han et al., "Lipin1 mediates cognitive impairment in fld mice via PKD-ERK pathway," Biochemical and Biophysical Research Communications, vol. 525, no. 2, pp. 286-291, 2020.

[10] R. N. Walsh and R. A. Cummins, "The open-field test: a critical review," Psychological Bulletin, vol. 83, no. 3, pp. 482-504, 1976.

[11] A. Ennaceur and J. Delacour, "A new one-trial test for neurobiological studies of memory in rats. 1: behavioral data," Behavioural Brain Research, vol. 31, no. 1, pp. 47-59, 1988.

[12] G. Levallet, M. Hotte, M. Boulouard, and F. Dauphin, "Increased particulate phosphodiesterase 4 in the prefrontal cortex supports 5-HT4 receptor-induced improvement of object recognition memory in the rat," Psychopharmacology, vol. 202, no. 1-3, pp. 125-139, 2009.

[13] R. Morris, "Developments of a water-maze procedure for studying spatial learning in the rat," Journal of Neuroscience Methods, vol. 11, no. 1, pp. 47-60, 1984.

[14] K. J. Livak and T. D. Schmittgen, "Analysis of relative gene expression data using real-time quantitative PCR and the 2 - $\Delta \Delta$ CT method," Methods, vol. 25, no. 4, pp. 402-408, 2001.

[15] Y. Zhou, Y. Luo, and J. Dai, “Axonal and dendritic changes are associated with diabetic encephalopathy in rats: an important risk factor for Alzheimer's disease," Journal of Alzheimer's Disease, vol. 34, no. 4, pp. 937-947, 2013.

[16] B. Cholerton, L. D. Baker, and S. Craft, "Insulin, cognition, and dementia," European Journal of Pharmacology, vol. 719, no. 1-3, pp. 170-179, 2013.

[17] S. Jimenez, M. Torres, M. Vizuete et al., "Age-dependent accumulation of soluble amyloid beta (Abeta) oligomers reverses the neuroprotective effect of soluble amyloid precursor protein-alpha (sAPP(alpha)) by modulating phosphatidylinositol 3-kinase (PI3K)/Akt-GSK-3beta pathway in Alzheimer mouse model," Journal of Biological Chemistry, vol. 286, no. 21, pp. 18414-18425, 2011.

[18] H.-C. Chiang, L. Wang, Z. Xie, A. Yau, and Y. Zhong, "PI3 kinase signaling is involved in Abeta-induced memory loss in Drosophila," Proceedings of the National Academy of Sciences of the United States of America, vol. 107, no. 15, pp. 70607065, 2010.

[19] H. Vlassara and M. R. Palace, "Glycoxidation: the menace of diabetes and aging," Mount Sinai Journal of Medicine, vol. 70, no. 4, pp. 232-241, 2003. 
[20] W. H. Gispen and G.-J. Biessels, "Cognition and synaptic plasticity in diabetes mellitus," Trends in Neurosciences, vol. 23, no. 11, pp. 542-549, 2000.

[21] B.-R. Li, Y.-J. Hsieh, Y.-X. Chen, Y.-T. Chung, C.-Y. Pan, and Y.-T. Chen, "An ultrasensitive nanowire-transistor biosensor for detecting dopamine release from living pc12 cells under hypoxic stimulation," Journal of the American Chemical Society, vol. 135, no. 43, pp. 16034-16037, 2013.

[22] M.-H. Liu, C. Yuan, J. He et al., "Resveratrol protects PC12 cells from high glucose-induced neurotoxicity via PI3K/Akt/ FoxO3a pathway," Cellular and Molecular Neurobiology, vol. 35, no. 4, pp. 513-522, 2015.

[23] R. Najafi, A. M. Sharifi, and A. Hosseini, "Protective effects of alpha lipoic acid on high glucose-induced neurotoxicity in PC12 cells," Metabolic Brain Disease, vol. 30, no. 3, pp. 731738, 2015.

[24] M. Farzaneh, Department of Pharmacology, Science and Research Branch, Islamic Azad University, Tehran, Iran, M. Sayyah et al., "The lentiviral vector pseudotyped by modified rabies glycoprotein does not cause reactive gliosis and neurodegeneration in rat hippocampus," Iranian Biomedical Journal, vol. 23, no. 5, pp. 324-329, 2019.

[25] A.-K. Kraeuter, P. C. Guest, and Z. Sarnyai, "The open field test for measuring locomotor activity and anxiety-like behavior," in Pre-Clinical Models Methods in Molecular Biology, P. Guest, Ed., vol. 1916, pp. 99-103, Humana Press, New York, NY, 2019.

[26] J. N. Crawley, "Exploratory behavior models of anxiety in mice," Neuroscience \& Biobehavioral Reviews, vol. 9, no. 1, pp. 37-44, 1985.

[27] T. L. Bale, A. Contarino, G. W. Smith et al., "Mice deficient for corticotropin-releasing hormone receptor-2 display anxietylike behaviour and are hypersensitive to stress," Nature Genetics, vol. 24, no. 4, pp. 410-414, 2000.

[28] M. Antunes and G. Biala, "The novel object recognition memory: neurobiology, test procedure, and its modifications," Cognitive Processing, vol. 13, no. 2, pp. 93-110, 2012.

[29] B. K. Goulart, M. N. M. de Lima, C. B. de Farias et al., "Ketamine impairs recognition memory consolidation and prevents learning-induced increase in hippocampal brain-derived neurotrophic factor levels," Neuroscience, vol. 167, no. 4, pp. 969-973, 2010.

[30] J. M. Silvers, S. B. Harrod, C. F. Mactutus, and R. M. Booze, "Automation of the novel object recognition task for use in adolescent rats," Journal of Neuroscience Methods, vol. 166, no. 1, pp. 99-103, 2007.

[31] J. Nunez, "Morris water maze experiment," Journal of Visualized Experiments, vol. 24, no. 19, 2008.

[32] R. Havekes, A. J. Park, J. C. Tudor et al., "Sleep deprivation causes memory deficits by negatively impacting neuronal connectivity in hippocampal area CA1," Elife, vol. 5, p. e13424, 2016.

[33] Q. Zhou, K. J. Homma, and M.-M. Poo, "Shrinkage of dendritic spines associated with long-term depression of hippocampal synapses," Neuron, vol. 44, no. 5, pp. 749757, 2004.

[34] M. B. Rust, "ADF/cofilin: a crucial regulator of synapse physiology and behavior," Cellular and Molecular Life Sciences, vol. 72, no. 18, pp. 3521-3529, 2015.

[35] R. C. Davis, I. T. Marsden, M. T. Maloney et al., "Amyloid beta dimers/trimers potently induce cofilin-actin rods that are inhibited by maintaining cofilin-phosphorylation," Molecular Neurodegeneration, vol. 6, no. 1, p. 10, 2011.

[36] G. M. Shankar, B. L. Bloodgood, M. Townsend, D. M. Walsh, D. J. Selkoe, and B. L. Sabatini, "Natural oligomers of the Alzheimer amyloid-beta protein induce reversible synapse loss by modulating an NMDA-type glutamate receptor-dependent signaling pathway," Journal of Neuroscience, vol. 27, no. 11, pp. 2866-2875, 2007.

[37] R. Niwa, K. Nagata-Ohashi, M. Takeichi, K. Mizuno, and T. Uemura, "Control of actin reorganization by slingshot, a family of phosphatases that dephosphorylate ADF/Cofilin," Cell, vol. 108, no. 2, pp. 233-246, 2002.

[38] S. Arber, F. A. Barbayannis, H. Hanser et al., "Regulation of actin dynamics through phosphorylation of cofilin by LIMkinase," Nature, vol. 393, no. 6687, pp. 805-809, 1998.

[39] S. J. Spratley, L. I. Bastea, H. Döppler, K. Mizuno, and P. Storz, "Protein kinase D regulates cofilin activity through p21activated kinase 4," Journal of Biological Chemistry, vol. 286, no. 39, pp. 34254-34261, 2011.

[40] T. Eiseler, H. Döppler, I. K. Yan, K. Kitatani, K. Mizuno, and P. Storz, "Protein kinase D1 regulates cofilin-mediated Factin reorganization and cell motility through slingshot," Nature Cell Biology, vol. 11, no. 5, pp. 545-556, 2009.

[41] W. Zhang, W. Zhong, Q. Sun, X. Sun, and Z. Zhou, “Adiposespecific lipin1 overexpression in mice protects against alcoholinduced liver injury," Scientific Reports, vol. 8, no. 1, p. 408, 2018.

[42] Z. Fang, S. Wang, X. Du, P. Shi, and Z. Huang, "Phosphatidate phosphatase- 1 is functionally conserved in lipid synthesis and storage from human to yeast," Acta Biologica Hungarica, vol. 65, no. 4, pp. 481-492, 2014.

[43] A. E. Mayer, M. C. Löffler, A. E. Loza Valdés et al., “The kinase PKD3 provides negative feedback on cholesterol and triglyceride synthesis by suppressing insulin signaling," Science Signaling, vol. 12, no. 593, p. eaav9150, 2019.

[44] K. Kolczynska, A. Loza-Valdes, I. Hawro, and G. Sumara, "Diacylglycerol-evoked activation of PKC and PKD isoforms in regulation of glucose and lipid metabolism: a review," Lipids Health Disease, vol. 19, no. 1, p. 113, 2020.

[45] M. C. Löffler, A. E. Mayer, J. Trujillo Viera et al., "Protein kinase D1 deletion in adipocytes enhances energy dissipation and protects against adiposity," The EMBO Journal, vol. 37, no. 22, 2018.

[46] A. Pasquier, K. Vivot, E. Erbs et al., "Lysosomal degradation of newly formed insulin granules contributes to $\beta$ cell failure in diabetes," Nat Commun, vol. 10, no. 1, p. 3312, 2019.

[47] L. Tarsa and Y. Goda, "Synaptophysin regulates activitydependent synapse formation in cultured hippocampal neurons," Proceedings of the National Academy of Sciences of the United States of America, vol. 99, no. 2, pp. 1012-1016, 2002.

[48] J.-Q. Wang, J. Yin, Y.-F. Song et al., "Brain aging and AD-like pathology in streptozotocin-induced diabetic rats," Journal of Diabetes Research, vol. 2014, Article ID 796840, 12 pages, 2014.

[49] G. T. Díaz-Gerevini, A. Daín, M. E. Pasqualini, C. B. López, A. R. Eynard, and G. Repossi, "Diabetic encephalopathy: beneficial effects of supplementation with fatty acids $\omega 3$ and nordihydroguaiaretic acid in a spontaneous diabetes rat model," Lipids in Health and Disease, vol. 18, no. 1, p. 43, 2019. 